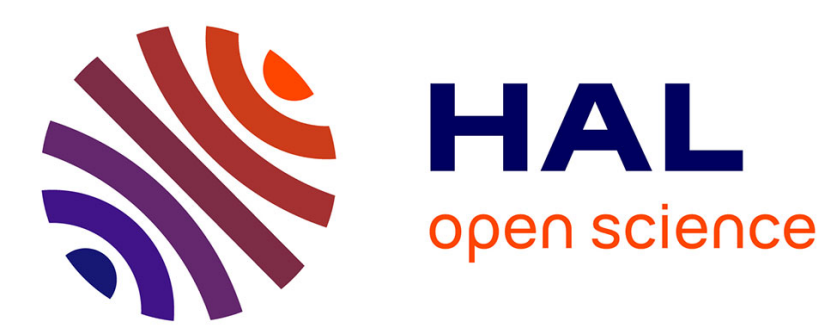

\title{
Des visas, pas de l'aide! De la migration comme substitut à l'aide au développement
}

\author{
Speranta Dumitru
}

\section{To cite this version:}

Speranta Dumitru. Des visas, pas de l'aide! De la migration comme substitut à l'aide au développement. Éthique publique - revue internationale d'éthique sociétale et gouvernementale , 2013, Enjeux éthiques de l'aide publique au développement, 15 (2), pp.77-98. 10.4000/ethiquepublique.1231 . hal01348298

\section{HAL Id: hal-01348298 \\ https://hal.science/hal-01348298}

Submitted on 31 Aug 2016

HAL is a multi-disciplinary open access archive for the deposit and dissemination of scientific research documents, whether they are published or not. The documents may come from teaching and research institutions in France or abroad, or from public or private research centers.
L'archive ouverte pluridisciplinaire HAL, est destinée au dépôt et à la diffusion de documents scientifiques de niveau recherche, publiés ou non, émanant des établissements d'enseignement et de recherche français ou étrangers, des laboratoires publics ou privés. 


\title{
DES VISAS, PAS DE L'AIDE! \\ DE LA MIGRATION COMME SUBSTITUT À L'AIDE AU DÉVELOPPEMENT ${ }^{1}$
}

\author{
SPERANTA DUMITRU \\ Université Paris Descartes et CERLIS, CNRS
}

Résumé: Si la migration est plus efficace que l'aide pour lutter contre la pauvreté, devrait-elle remplacer l'aide? Pas toujours. Cet article propose un critère pour distinguer les cas où la migration doit se substituer à l'aide au développement, des cas où elle doit en être le complément. Selon ce critère, un programme du développement est «pauvreté-efficace » lorsqu'il conduit à sortir le plus grand nombre des personnes de la pauvreté. Par conséquent, un tel programme cherchera toujours à compléter l'aide par d'autres politiques conduisant à sortir de la pauvreté des personnes additionnelles. En revanche, lorsque l'aide n'est pas efficace, elle devra être abandonnée et remplacée par des politiques migratoires et d'autres mesures efficaces pour combattre la pauvreté.

En 2006, la Banque mondiale a recommandé aux pays riches d'envisager l'ouverture de leurs frontières comme une politique de développement. Elle a calculé qu'une une migration qui viendrait augmenter de $3 \%$ la population active des pays riches pourrait apporter aux pays pauvres

1. Cette recherche a été réalisée avec le soutien de la Fondation des Sciences sociales. L'auteure remercie tout particulièrement François Héran, Caroline Axelle Caplan, Caroline Guibet Lafaye, Olivier Nalin, Solange Chavel, Gaëlle Demelemestre, Patrick Pharo, ainsi que Vanessa Barbé, MarieLaure Basilien Guinche, Géraldine Bozec, Gilles de Margerie, Stéphane Dufoix, Angéline EscafréDublet, Jérémie Gauthier, Anne-Sophie Lamine, Catherine Le Bris, Virginie Silhouette-Dercourt, Daniel Sabbagh, Patrick Simon, les éditeurs et les deux évaluateurs anonymes pour leur lecture, commentaires et suggestions. 
l'equivalent de quatre fois et demie l'aide au développement (Banque mondiale, 2006; Walmsley et Winters, 2003). D’autres études le confirment. Cette augmentation de $3 \%$ représente le double de ce que les pays pauvres pourraient gagner non pas d'une mais de trois politiques de développement conjuguées : l'aide publique au développement, la libéralisation totale du commerce mondial et l'annulation de la dette (Pritchett, 2006).

Devant ces comparaisons, on ne saurait éviter une question. Si une modeste augmentation de la migration apporte plusieurs fois le volume de l'aide, ne faudrait-il pas changer de politique ? Les acteurs du développement ne devraient-ils pas défendre la migration de travail, en exigeant «des visas, pas de l'aide», tout comme certains demandent « du commerce, pas de l'aide»? On pourrait penser que la bonne formule n'est pas «des visas, pas de l'aide ", mais « des visas et de l'aide ", qui suggère que la migration est un complément, non un substitut de l'aide au développement. Or, la thèse que l'on soutient ici est qu'il existe des cas où la migration doit se substituer à l'aide au développement.

Le but du présent article est de proposer une méthode pour distinguer les cas où la migration doit se substituer à l'aide au développement, des cas où elle doit en être le complément. Cette méthode s'inspire du critère de "pauvreté-efficacité » proposé par Paul Collier et David Dollar (2002). Ces économistes ont soutenu que l'aide doit être distribuée entre différents pays de façon à pouvoir sortir le plus grand nombre de personnes de la pauvreté. Le présent article propose d'étendre ce critère audelà de l'aide, à l'évaluation de politiques de développement plus complexes, composées de plusieurs mesures. On pourra ainsi montrer que dans les pays où l'aide ne combat pas efficacement la pauvreté, elle doit être remplacée par d'autres mesures, telles les politiques migratoires; refuser de le faire, c'est piéger les personnes dans leurs États et dans la pauvreté. On montrera également que dans les pays où l'aide est pauvretéefficace, la migration doit être envisagée comme un complément de l'aide.

L'article est divisé en cinq sections. Les deux premières sections visent à clarifier la notion de "piège à pauvreté » auquel l'aide au développement est censée répondre, ainsi que les recherches sur l'efficacité de l'aide. C'est dans la troisième section que le critère de pauvreté-efficacité est présenté et généralisé pour montrer la nécessité d'intégrer les politiques migratoires dans un programme de lutte contre la pauvreté. La quatrième 
section explique pourquoi la reconnaissance de la migration comme levier du développement a eu tant de peine à s'imposer. La dernière section souligne les conséquences de l'adoption du critère de pauvreté-efficacité. Ce critère est ambitieux: il nous demande de choisir la politique qui maximise le nombre de personnes sorties de la pauvreté. Or, si la libéralisation complète de la mobilité combat mieux la pauvreté, comme certaines études semblent l'indiquer, notre critère nous recommandera d'adopter cette politique plutôt que de limiter l'augmentation de la migration de travail à $3 \%$.

\section{LE PIÈGE À PAUVRETÉ}

La difficulté d'envisager des substituts à l'aide au développement repose sur la croyance que l'aide est une solution au piège à pauvreté. Qu'est-ce donc qu'un piège à pauvreté ? Et surtout, l'aide au développement est-elle la solution incontournable pour en sortir?

L'économiste William Easterly (2006), qui a fait connaître au grand public les recherches sur l'efficacité de l'aide, pense que non. De manière quelque peu malhabile, il a présenté son analyse comme une critique de la notion de piège à pauvreté, qui ne serait qu'un «mythe » et motiverait à tort des économistes comme Jeffrey Sachs à demander inlassablement une augmentation de l'aide. Conseiller aux Nations Unies pour les objectifs du Millénaire, Sachs soutient effectivement que les pauvres se retrouvent piégés par la pauvreté et que pour en sortir,

[1]a solution est [...] l'aide extérieure, sous la forme de l'aide au développement, [qui] permet de démarrer le processus d'accumulation du capital, de croissance économique et d'élever le revenu des ménages. L'aide extérieure nourrit trois dimensions. Un tout petit peu va directement vers les ménages, principalement pour les urgences humanitaires comme l'aide alimentaire pendant une sécheresse. Beaucoup plus va directement au budget pour financer les investissements publics et une partie est dirigée vers le secteur privé (par exemple, vers les agriculteurs) au travers des programmes de microcrédits [...]. Si l'aide extérieure est suffisamment substantielle et se maintient suffisamment longtemps, le capital augmente suffisamment pour élever les ménages au-dessus de la subsistance. À ce point, le piège à pauvreté est brisé [...] la croissance 
s'auto-entretient au travers de l'épargne des ménages et des investissements publics soutenus par l'imposition des ménages. En ce sens, l'aide extérieure n'est pas une distribution de prestations sociales, mais un investissement qui brise les pièges à pauvreté une fois pour toutes $(2005: 246)^{2}$.

Easterly est conscient que ni le problème (le «piège à pauvreté ») ni la solution proposée (l'aide extérieure) ne sont l'invention de Sachs. En effet, l'histoire de la notion de piège à pauvreté dans l'économie du développement remonte au moins aux années 1950 lorsqu'elle était discutée sous le nom de «cercle vicieux de la pauvreté » (Winslow, 1951; Myrdal, 1957 ; Nurkse, 1953). Mieux que l'idée de piège, celle de cercle vicieux suggère le lien causal par lequel la pauvreté produit de la pauvreté. Comme l'indiquait Ragnar Nurkse,

ce concept implique un faisceau circulaire des forces qui tendent à agir et réagir l'une sur l'autre de sorte qu'elles maintiennent un pays pauvre dans un état de pauvreté. Il n'est pas difficile d'imaginer une telle circularité. Par exemple, il est probable qu'un pauvre n'a pas assez à manger; souffrant de malnutrition, sa santé s'affaiblit ; étant physiquement faible, il a une faible capacité à travailler; cela implique qu'il sera pauvre, qu'il n'aura pas assez à manger et ainsi de suite. Une telle situation, appliquée à un pays pris comme un tout, peut être résumée par la proposition banale « un pays est pauvre parce qu'il est pauvre» (1953: 4).

L'idée que pour sortir du cercle vicieux de la pauvreté, il est nécessaire d'injecter du capital est, elle aussi, ancienne. Elle est proposée, pendant la guerre, par Paul Rosenstein-Rodan, le père de la théorie du grand coup de pouce (big push). Selon lui, une aide extérieure était nécessaire pour financer l'industrialisation des «régions internationales défavorisées » (comme il qualifiait alors l'Europe de l'Est et du Sud) et promouvoir ainsi la «maximisation du revenu mondial» (Rosenstein-Rodan, 1943 : 202). Cette idée est aussi suggérée par les premiers modèles qui essayent, avant la guerre, d'expliquer et de formaliser l'absence de croissance économique. Le premier, le modèle Harrod-Domar, du nom des deux économistes

2. Toutes les traductions sont de l'auteure. 
keynésiens, explique l'absence de croissance par un déficit d'épargne. Dans les pays pauvres ou en récession, il existe un écart entre l'offre de travail, qui se trouve en excès, et l'absence de capital, qui permettrait de l'employer. Normalement, l'épargne privée ou l'investissement public comblent cet écart, mais quand ces deux sources de capital sont déficitaires, comme c'est le cas dans les pays pauvres, l'aide extérieure est nécessaire. Un second modèle à deux déficits (dual gap) ajoute au déficit d'épargne le déficit en devises (Chenery et Strout, 1966). Les pays pauvres, n'étant pas compétitifs et ne retirant pas assez de revenus des exportations, ont un déficit de devises qui peut être compensé par une aide extérieure. Enfin, un modèle à trois déficits ajoute le déficit fiscal aux deux insuffisances déjà mentionnées, en arguant de l'incapacité des pays pauvres à lever des impôts pour financer l'investissement (Taylor, 1990).

Easterly critique ces théories. Il soutient, d'une part, que l'aide n'est pas efficace pour répondre à la pauvreté et, d'autre part, que le piège n'est pas seulement un piège à pauvreté, d'autres facteurs étant responsables de la stagnation dans la pauvreté. C'est en ce sens qu'il affirme que le piège à pauvreté est un mythe, en soulignant que des facteurs autres que la pauvreté elle-même contribuent au piège. Certaines recherches sur l'efficacité de l'aide lui donnent en partie raison.

\section{L'AIDE AU DÉVELOPPEMENT AIDE-T-ELLE LE DÉVELOPPEMENT?}

Les modèles de croissance prédisant que l'aide extérieure permet de sortir du piège à pauvreté ont commencé à être testés dans les années $1970^{3}$. Les premières études ont été surprenantes. Elles ont établi une corrélation négative entre l'entrée des capitaux et l'augmentation de l'épargne, à l'inverse de ce que prédisait le modèle Harrod-Domar (Griffin, 1970). Certains économistes ont alors essayé d'expliquer l'absence d'épargne par le fait que la consommation était encouragée par les donateurs; ils ont refait le calcul en désagrégeant les flux de capitaux (en aide extérieure, investissement étranger et autres flux) et ont trouvé une corrélation, cette fois

3. Je suis ici en partie la revue de littérature faite par McGillivray (2006). 
positive, entre aide et épargne (Papanek, 1973). Mais d'autres économistes, avec des données plus nombreuses et collectées dans un plus grand nombre de pays pour l'ensemble de la décennie 1970, ont trouvé une corrélation négative entre aide extérieure et croissance (Mosley, 1980).

Jusqu'à la fin des années 1990, aucun consensus ne s'est dégagé sur l'effet bénéfique ou nuisible de l'aide. Certaines revues de littérature indiquaient que « de manière surprenante, nous savons très peu de choses sur l'impact macroéconomique de l'aide » et expliquaient que «la pauvreté de la théorie, combinée à la faiblesse de la méthodologie économétrique, rend difficile de conclure quoi que ce soit sur la relation entre l'aide et l'épargne ou entre l'aide et la croissance» (White, 1992: 121).

Un consensus sur l'efficacité de l'aide a commencé à émerger après la publication du rapport de la Banque mondiale (1998) L'évaluation de l'aide, ce qui marche, ce qui ne marche pas et pourquoi. Ce consensus s'établit autour de l'idée que l'aide peut avoir un effet bénéfique à condition d'être accompagnée par une «bonne gouvernance». Le rapport de la Banque mondiale s'est principalement appuyé sur les résultats de Craig Burnside et David Dollar (2000), mais l'idée de tester la corrélation entre les flux de l'aide et les politiques des pays destinataires était déjà venue à Peter Boone (1996). Celui-ci avait choisi d'évaluer le lien entre l'aide et des variables plus politiques, y compris les indicateurs du développement humain. En analysant les données d'un nombre important de pays sur deux décennies, il avait établi que l'aide extérieure augmentait la consommation publique et la taille du gouvernement, mais ne bénéficiait ni à l'investissement ${ }^{4} \mathrm{ni}$ aux pauvres. Selon ses calculs, bien que la consommation augmente, l'aide n'a aucun effet significatif sur l'amélioration des indicateurs du développement humain (taux de scolarisation, espérance de vie). Son explication est que l'aide bénéficie principalement à l'élite au pouvoir. Si le caractère démocratique du régime n'influe pas sur l'efficacité de l'aide, Boone constate que dans les démocraties libérales, la mortalité infantile est, toutes choses égales d'ailleurs, d'un tiers plus basse que dans les régimes autoritaires.

Les recherches de Burnside et Dollar (2000), sur lesquelles s'est appuyé le rapport de la Banque mondiale, montrent que l'aide peut avoir un effet bénéfique seulement dans un certain environnement politique.

4. Sauf dans les pays de taille réduite ou avec un ratio aide/PIB important (Boone, 1996).

ÉTHIQUE PUBLIQUE 
Par environnement politique, ils entendent les politiques fiscales, monétaires et commerciales qui influent sur la croissance. L'aide a un effet bénéfique dans des pays qui maintiennent une inflation basse, qui ont des déficits budgétaires réduits et qui sont ouverts au commerce international. Toutefois, les deux économistes découvrent que l'aide est rarement allouée aux pays en fonction de la qualité des politiques que l'on y mène et observent que l'aide bilatérale conduit davantage à l'augmentation des dépenses. Selon eux, pour améliorer l'efficacité de l'aide, celle-ci devrait être envoyée en priorité aux pays qui mènent de bonnes politiques. C'est une idée que développeront Collier et Dollar (2002) et que nous discuterons dans la prochaine section.

Deux conclusions peuvent être tirées de ces résultats. La première est que Easterly semble avoir raison: les plus pauvres du monde ne sont pas piégés uniquement par la pauvreté. La seconde conclusion, plus importante, est que les pauvres peuvent aussi être piégés par les politiques menées dans leurs propres États.

\section{LES ÉTATS, PIÈGES À PAUVRETÉ?}

Collier et Dollar (2002) ont eu l'idée de mesurer l'efficacité de l'aide par le nombre de personnes qu'elle sort de la pauvreté. Ils ont comparé l'allocation existante de l'aide à une allocation qui privilégierait les pays avec une bonne gouvernance et ont calculé que celle-ci permettrait de sortir de la pauvreté quelque 18 millions de personnes additionnelles chaque année. Ils ont alors plaidé pour un changement de politique sur la base d'un critère qu'ils ont appelé "pauvreté-efficacité ». L'aide est dépensée de façon pauvreté-efficace quand sa distribution entre les pays conduit à maximiser le nombre de personnes sorties de la pauvreté.

Nous proposons ici d'étendre le critère de pauvreté-efficacité au-delà du cadre de l'allocation de l'aide entre les pays pour lequel il a été élaboré. Dès lors, on définira une politique de développement pauvreté-efficace comme l'ensemble de mesures qui, combinées, permettent de sortir le plus grand nombre de personnes de la pauvreté. Cette extension du critère permet de démontrer deux thèses. La première, qu'on peut appeler de "l'incomplétude de l'aide», soutient que l'aide à elle seule ne peut pas constituer une politique de développement pauvreté-efficace. On peut 
montrer cela pour tout niveau positif d'efficacité de l'aide. En effet, même dans les pays où l'aide est efficace, s'il existe une seule autre mesure capable de sortir des personnes additionnelles de la pauvreté, cette mesure doit être considérée comme un complément de l'aide par toute politique pauvretéefficace. La raison est que par définition, une politique pauvreté-efficace vise à maximiser le nombre de personnes sorties de la pauvreté. Or, de telles mesures sont nombreuses et la politique migratoire en fait partie, comme nous le montrerons dans la section suivante. Si tel est le cas, refuser d'intégrer la migration à l'agenda du développement revient à choisir une politique de développement moins pauvreté-efficace. La seconde thèse peut être appelée la «la substitution de l'aide». Quand l'aide n'est pas efficace, refuser de considérer la migration comme un substitut de l'aide, c'est refuser de mener une politique de développement tout court, lorsque par «politique de développement » on entend une action de lutte contre la pauvreté. Le critère de pauvreté-efficacité étendu permet donc de déterminer les cas où la devise « des visas, pas de l'aide » s'applique, à savoir les cas où l'aide n'a pas d'effet ou a un effet nuisible sur la pauvreté. Il permet aussi de distinguer cette situation de tous les autres cas où c'est la devise «des visas et de l'aide» qui est la mieux adaptée.

Le critère de pauvreté-efficacité montre qu'une politique exclusivement centrée sur l'aide ne combat pas efficacement la pauvreté. Mais il suggère aussi qu'une telle politique conduit à "piéger» les pauvres dans leurs États. On peut montrer cela en revenant aux recommandations de Collier et Dollar (2002), mentionnées dans la section précédente. En effet, une allocation de l'aide pauvreté-efficace recommande d'orienter l'aide vers les pays où elle réussit le mieux à combattre la pauvreté et d'éviter les pays où elle n'a que peu ou aucun effet en ce sens. À première vue, ce tri entre les pays semble difficile à accepter, car il semble désavantager des millions de personnes qui non seulement sont pauvres, mais qui également ont la malchance de vivre dans des pays mal gouvernés. Or, à y regarder de plus près, le mode d'allocation de l'aide ne les désavantage pas. Si, dans un pays donné, un dollar d'aide n'améliore le sort d'aucun pauvre, envoyer ce dollar au pays voisin où il a de l'effet n'est pas une action qui désavantage les pauvres du premier pays. Ces personnes sont certes désavantagées, mais l'aide n'y peut rien. Ce qui désavantage les pauvres, c'est notre croyance que leur seule option passe par l'aide accordée aux États. Refuser d'envisager 
des substituts à l'aide lorsqu'elle n'est pas efficace, revient à piéger les pauvres dans les États où ils sont nés.

Une politique de développement centrée sur l'aide peut piéger les pauvres de deux façons: elle peut les enferrer dans le cercle vicieux de la pauvreté, comme elle peut les piéger, dans un sens spatial du mot piège. Premièrement, le «cercle vicieux de la pauvreté» peut être renforcé en accordant aux États le rôle de protagonistes du développement. Les États, principaux administrateurs de l'aide, se reconnaissent mutuellement comme protagonistes du développement quelle que soit leur réussite effective à combattre la pauvreté. En signant des accords bilatéraux ou internationaux, ils maintiennent et renforcent leur suprématie. Or, pour les pauvres qui vivent dans des États peu à même d'améliorer leur sort, le renforcement de la suprématie des États agit comme un piège : il maintient la pauvreté tout en éloignant la perspective que de nouveaux acteurs du développement apportent de meilleures solutions. L'étatisation excessive du développement (par une politique centrée sur l'aide, administrée par les États) présente un risque de perpétuer le cercle vicieux de la pauvreté.

Nous devons toutefois éviter les généralisations hâtives. Il est vrai que certains économistes, s'adressant à un large public, ont fait valoir l'existence d'un «piège de l'aide» (Hubbard et Duggan, 2009) ou d'un «cercle vicieux de l'aide» qui "tarit les investissements, crée une culture de la dépendance et fait le jeu d'une corruption illimitée» (Moyo, 2009: 95). D'autres études confirment que l'aide encourage l'élite politique à développer des comportements de recherche de rente et nous pouvons ajouter qu'un système international qui fait des États les principaux acteurs renforce cette rente. De même, on a pu montrer que statistiquement, les gouvernements les plus corrompus reçoivent plus d'aide que ceux qui sont moins corrompus (Alesina et Weder, 2002). Cependant, tous les États donateurs ne se comportent pas de la même manière: si les États-Unis, par exemple, semblent donner plus d'aide aux pays corrompus, les pays scandinaves sont plus généreux avec les pays moins corrompus ${ }^{5}$. Des

5. Alesina et Weder (2002) montrent que si la situation est plus contrastée par rapport à l'aide bilatérale, l'aide multilatérale est insensible à la corruption, y compris après la diffusion de la rhétorique de la «bonne gouvernance». À comparer toutefois à d'autres études, comme Tavares (2003) qui montre que l'aide fait décroître la corruption. 
études plus approfondies sont donc nécessaires pour étayer le risque que l'étatisation excessive du développement perpétue la pauvreté.

Deuxièmement, on peut dire qu'une politique centrée sur l'aide piège les pauvres à partir du sens spatial du mot piège. Il a déjà été relevé que la construction des États conduit à "emprisonner» les populations (Mann, 1993) ou à les «étreindre» entre leurs frontières (Torpey, 2001). Ces analyses acquièrent une nouvelle signification à la lumière des recherches sur l'efficacité de l'aide. Celles-ci soulignent parfois l'importance des facteurs territoriaux. Par exemple, certaines études ont montré que l'efficacité de l'aide dépend des conditions climatiques (Guillaumont et Chauvet, 2001). D'autres études montrent que la situation enclavée d'un pays (sans accès à la mer) ou l'abondance des ressources naturelles sont des pièges à pauvreté (Collier, 2008). De même, l'instabilité politique a été analysée comme cause de l'inefficacité de l'aide, celle-ci n'ayant aucun effet sur la croissance des pays en proie aux changements politiques violents (Islam, 2005 ; Collier, 2008). L'objectif de ces études était de contester l'explication de l'inefficacité de l'aide par la seule mauvaise gouvernance. Mais elles soulèvent indirectement la question suivante: faut-il piéger les pauvres dans des régions enclavées, mal gouvernées, avec des conditions climatiques défavorables ou des conflits? Considérer la migration comme un complément ou une solution de remplacement à l'aide permet de sortir d'un piège à pauvreté territorial.

\section{LA MIGRATION COMME DÉVELOPPEMENT}

Que la mobilité constitue un facteur de prospérité est l'un des faits les moins controversés chez les économistes. John Kenneth Galbraith s'étonnait de la difficulté à faire accepter cette idée :

La migration est le plus ancien moyen de lutte contre la pauvreté. Elle sélectionne ${ }^{6}$ ceux qui souhaitent l'aide au plus haut point. Elle est bénéfique pour le pays où ils se rendent; elle aide à briser l'équilibre de la pauvreté de leurs pays d'origine. Quelle est alors cette perversité de l'âme humaine qui pousse les gens à résister à un bien aussi manifeste? $(1979: 149)$

6. En économie, le mot sélection doit être entendu comme «auto-sélection» et non comme le résultat d'un processus de sélection des individus mis en place par le pays hôte. 
En effet, pendant les années 1970 et 1980, l'idée que la migration puisse être un levier de développement apparaissait comme incongrue aux acteurs du développement. Ces décennies, dominées par les perspectives structuralistes et néomarxistes sur le développement, imposent l'idée que la migration est le résultat néfaste de l'expansion du capitalisme. La migration est analysée comme n'importe quel autre flux manipulé par le capital international pour atteindre ses buts expansionnistes; les migrants sont vus comme de simples pions, dont les actions dépendent de forces qu'ils ne contrôlent pas ${ }^{7}$. On soutient que la migration les déracine, disloque leurs vies et détruit les traditions. La migration ne peut qu'aggraver les inégalités entre le centre et la périphérie, elle produit le «développement du sous-développement» et prive les pays pauvres du peu de capital humain qu'ils possèdent ${ }^{8}$. Cette période coïncide avec le débat sur la « fuite des cerveaux " réinstallé comme combat mené au nom des pays pauvres ${ }^{9}$. Les organisations internationales engagées dans le développement suivent cette représentation des migrants. La Conférence des Nations Unies sur le commerce et le développement (CNUCED) désigne les migrants diplômés par le curieux syntagme «transfert inverse de technologie » pour signifier que les bénéfices de l'aide technologique sont réinjectés dans les pays industrialisés lorsque le personnel qualifié immigre. Chaque année entre 1974 et 1987, la CNUCED publie des rapports sur le «transfert inverse de technologie» sans mentionner le mot migrant ${ }^{10}$. De façon générale, les migrations sont déshumanisées: comprises du point de vue étatique, elles sont décrites comme de l'exportation, du pillage ou de l'échange de main-d'œuvre; elles contrarient les politiques du développement.

Un fonctionnaire du développement qui se serait endormi dans les années 1970 pour se réveiller dans les années 2000 aurait du mal à comprendre que les organisations internationales publient aujourd'hui des rapports intitulés Lever les barrières. Mobilité et développement bumain

7. Pour une description, voir Jones (1998).

8. Pour une description de ces conceptions, voir Haas (2010).

9. Le débat sur la fuite des cerveaux est apparu comme un conflit anglo-américain qui s'est étendu aux autres pays riches. Le changement de la loi de l'immigration aux États-Unis, qui privilégiait les ressortissants européens, a ouvert les frontières aux ressortissants non européens et a «moralisé » le débat sur la fuite des cerveaux. Voir Dumitru (2009) sur ce sujet.

10. Voir la bibliographie établie par Gaillard et Gaillard (1998). 
(PNUD, 2009) et que certains États s'incitent réciproquement à considérer les migrants comme des "acteurs du développement» (Déclaration de Dhaka, 2013). Ce fonctionnaire, apprenant que l'Assemblée générale des Nations Unies a organisé en 2006 et 2013 deux Dialogues de haut niveau sur le rapport entre migration et développement, serait étonné de constater que certains acteurs évoquent l'intégration de la migration à l'agenda du développement qui succédera en 2015 aux Objectifs du Millénaire. S'il existe aujourd'hui des raisons d'être pessimiste par rapport à cette intégration que les États sont appelés à voter, il n'est pas moins vrai qu'un changement important a eu lieu au sein des organisations internationales chargées du développement. Qu'est-ce qui explique ce changement?

On peut identifier deux facteurs qui ont favorisé ce changement. Le premier est le changement de "philosophie» en matière de développement. Jusqu'à la fin des années 1980, le développement est compris comme un changement macroéconomique qui se traduit en termes de croissance et s'exprime par des données agrégées à l'échelle nationale (PIB, revenu moyen, etc.). À partir de 1990, une nouvelle conception du développement apparaît et s'institutionnalise par le rapport annuel du Programme des Nations Unies pour le développement (PNUD) sur le développement humain. L'économiste Mahbub U1 Haq, à l'origine de ce rapport, s'inspire de l'approche des capabilités d'Amartya Sen lorsqu'il soutient que ce qui compte en dernière instance dans le développement, c'est la personne humaine et l'élargissement de ses capacités d'agir. L'indicateur du développement humain lancé par le PNUD en 1990 est destiné à approximer l'élargissement de la sphère d'options individuelles au moyen de trois variables : la santé (l'espérance de vie à la naissance), l'éducation (le taux d'analphabétisme) et le niveau de revenu. Si ces données, que les États sont invités à collecter chaque année, restent agrégées et rendent difficilement compte de la liberté individuelle, l'adoption de l'approche des capabilités opère un changement. La vision macroéconomique et stato-centrée du développement fait place à une conception centrée sur la personne humaine et ses possibilités d'action. Ce changement de philosophie favorise, à notre avis, la réorientation des rapports entre migration et développement. Comme le développement concerne les capacités d'agir des individus et comme la mobilité en fait partie, il devient aisé de soutenir 
que la migration, une sous-catégorie de la mobilité, favorise le développement humain (PNUD, 2009).

Le second facteur expliquant la transformation du rapport entre migration et développement est l'accumulation d'études empiriques, notamment sur les envois de fonds des migrants. En effet, ceux-ci connaissent une augmentation vertigineuse depuis la fin des années 1980: de 15 fois en 20 ans. Toutefois, l'argument des transferts financiers n'aurait pas suffi à changer la perception de la migration, car selon les conceptions structuralistes et néomarxistes, ces flux monétaires sont censés avoir des effets négatifs. En particulier, ils sont présumés accroître les inégalités (favorisant les familles des migrants considérées comme plus aisées) et décroître la productivité locale (car les bénéficiaires sont censés acheter des produits de consommation d'importation). Pourtant, dès les années 1970 quelques études empiriques montraient déjà le contraire. Ainsi, on avait documenté des transferts d'argent arrivant dans des régions rurales pauvres du tiers-monde (au Mexique, aux Antilles, en Anatolie), voire du premier monde (le sud de l'Espagne des années 1970). À la différence de l'aide qui arrivait rarement dans ces régions, les envois d'argent des migrants conféraient du pouvoir aux communautés locales éloignées, en leur offrant un moyen de survie indépendant de l'aléa politique.

L'amélioration des outils statistiques a permis de consolider ces résultats. Les chiffres sont parlants. Au total, les envois d'argent représentent aujourd'hui quatre fois l'aide au développement, plus de 500 milliards de dollars envoyés annuellement. Autrement dit, les migrants du Sud qui représentent 2,2\% de la population mondiale et qui, pour moitié, résident dans d'autres pays du $S^{11} d^{11}$ réussissent à transférer des montants quatre fois supérieurs aux montants consentis par les États. Dans certains pays, les transferts d'argent constituent un tiers du PIB et parfois la moitié ${ }^{12}$. Ils contribuent à baisser la pauvreté. Avec des données collectées dans

11. Selon les données publiées en septembre 2013 par le DAES des Nations Unies, le nombre de migrants internationaux est de 232 millions, ce qui représente 3,2\% de la population mondiale. Les migrants du Sud qui résident dans les pays du Nord représentent 81,9 millions de personnes, moins que les migrants originaires du Sud résidant dans un pays du Sud, qui sont au nombre de 82,3 millions.

12. Selon la Banque mondiale, les pays où les envois d'argent comme part du PIB sont les plus importants sont le Tadjikistan (47\%), le Liberia (31\%), la République kirghize (29\%), le Lesotho (27\%), la Moldavie (23\%), le Népal (22\%). 
81 pays, Richard Adams et John Page (2005) établissent qu’une croissance de $10 \%$ des envois d'argent correspond à une baisse moyenne de la proportion des personnes vivant dans la pauvreté de 3,5\%; de même, une croissance de $10 \%$ de la proportion d'émigrés dans la population d'un pays conduit à un déclin moyen de 2,1\% de la part des personnes vivant dans ce même pays avec moins de un dollar par jour. L'argent des migrants n'est pas utilisé que pour la consommation: les ménages investissent dans l'éducation, dans la construction de logement. Ces montants ne bénéficient donc pas seulement à leurs familles. L'argent des migrants a un effet bénéfique sur la croissance (Faini, 2002). Il a un effet multiplicateur sur le développement des pays : au Mexique, par exemple, on a calculé que chaque dollar envoyé générait entre 2,69 et 3,17 dollars, selon les ménages qui le recevaient (Adelman et Taylor, 1990) et chaque 2 milliards de dollars reçus augmentait la production de 6,5 milliards (Durand et al., 1996).

Les États eux-mêmes ont compris l'importance de l'argent envoyé par les migrants. Les envois de fonds sont un flux financier qui s'avère des plus stables et sûrs, quelle que soit la conjoncture économique (Sirkeci $e t$ al., 2012). Certains États ont ainsi commencé à les utiliser comme gage pour des emprunts étatiques, tandis que d'autres émettent des obligations en empruntant à leurs diasporas (par la création des diaspora bonds). Bien que les envois de fonds des migrants relèvent souvent d'un choix privé ayant pour destination leurs familles, leurs conséquences vont au-delà du bien-être de ces familles. De même, de nombreuses associations diasporiques sont créées pour mener des projets de développement dans les pays d'origine.

Deux remarques doivent être ajoutées à cette brève analyse qui ne saurait être exhaustive. La première remarque est que le rapport entre migration et développement ne se réduit pas à la valeur des flux financiers. Par exemple, une importante littérature a émergé sur le concept de transferts sociaux (social remittances), forgé par Peggy Levitt (1998). Ce concept désigne l'ensemble d'idées, de normes, de pratiques que les migrants transfèrent vers les pays d'origine et qui ont un rôle important dans le développement. La seconde remarque est que le couple migrationdéveloppement ne se réduit pas aux conséquences que la migration a sur les pays d'origine ou de destination. En réaction à cette tendance, Michael 
Clemens et Lant Pritchett ont souligné que «ce sont les gens, et non les bouts de terre, qui éprouvent du bien-être » (2008: 395). Ils ont critiqué l'anomalie qui caractérise les statistiques du développement et qui consiste à évaluer les phénomènes uniquement à l'intérieur des frontières nationales. Par exemple, tandis qu'une augmentation de revenu de $30 \%$ qu'un Salvadorien obtient en allant travailler à la ville est incluse dans les statistiques, la multiplication de son revenu par cinq lorsqu'il se rend travailler au Texas ne laissera aucune trace dans les statistiques du développement, si ce n'est que par les envois d'argent. Clemens et Pritchett forgent alors le concept de revenu per natural pour désigner le revenu moyen des personnes nées dans un pays donné, quel que soit leur lieu de résidence. Sans surprise, ce revenu augmente avec le nombre des migrants et avec la différence de revenu moyen entre le pays de naissance et le pays de résidence.

\section{DES VISAS, PAS... DE LA LIBRE CIRCULATION ?}

Nous avons montré que le critère de pauvreté-efficacité permet de déterminer les cas où la devise « des visas, pas de l'aide » est appropriée et les cas où la devise «des visas et de l'aide » s'applique. Pritchett, qui recommandait une augmentation de $3 \%$ de la migration de travail, adoptait une position pondérée, sans envisager l'abandon de l'aide. Il avait d'ailleurs écrit :

Combien de temps encore les aides financières seront-elles les seuls mécanismes pour promouvoir le développement? Combien de temps seuls la Bolivie, l'Arménie ou le Nigéria doivent figurer à l'ordre du jour international et non les Boliviens, les Arméniens ou les Nigérians (2006: 140).

Cependant, le critère de pauvreté-efficacité est un critère ambitieux : il nous demande de choisir la politique qui maximise, et non qui augmente, le nombre de personnes sorties de la pauvreté. Si une autre mesure peut sortir plus de personnes de la pauvreté, le critère de pauvretéefficacité nous demande de la prendre. Cette mesure n'est-elle pas la libre circulation?

En effet, pour montrer que la libre circulation combat efficacement la pauvreté, on peut avoir recours aux études historiques ainsi qu'aux modèles formels. Les études portant sur la période 1840-1919 sont utiles 
pour plusieurs raisons. La première est que cette période connaît une intense circulation internationale qui prendra fin après la Première Guerre mondiale, lorsque le nationalisme rend obligatoire le contrôle des passeports $^{13}$. La deuxième raison est qu'à la différence des périodes antérieures où la circulation internationale n'était pas non plus contrôlée par des passeports, le XIX ${ }^{\mathrm{e}}$ siècle a l'avantage de fournir des données relativement fiables. La troisième raison est que ces données sont suffisamment fiables pour établir une corrélation positive entre une circulation internationale intense et le développement économique. Appelée aussi «l'âge de la migration de masse », la période 1840-1919 a connu un taux important et croissant de migrants européens qui traversent l'Atlantique ${ }^{14}$. Au début du $\mathrm{XX}^{\mathrm{e}}$ siècle, ce taux est de un million de migrants par an. On estime qu'ils furent plus de 55 millions pour toute la période, un volume important si on le compare à la démographie européenne de l'époque ou au volume des migrants d'aujourd'hui (232 millions, toutes origines confondues). Ces migrations sont poussées par la famine (la crise irlandaise de la pomme de terre), par les impôts et la croissance démographique; elles sont favorisées par la baisse des coûts des transports transatlantiques. Elles prendront fin avec la Grande Guerre, qui installe le monopole des moyens de contrôle de la circulation par les États, tel que nous le connaissons aujourd'hui (Torpey, 2001).

La période de la grande migration a été une période d'intense croissance économique. Mieux, elle a permis aux pays les moins avancés de rattraper le développement des pays les plus avancés, à la fois en Europe et entre l'Europe et le Nouveau Monde. On appelle ce processus la « convergence », en référence à la diminution des inégalités entre les pays. Timothy Hatton et Jeffrey Williamson (2005) ont évalué la convergence mesurée en termes de salaires ajustés en parité de pouvoir d'achat et ont établi que la migration est responsable de l'augmentation de la convergence jusqu'à la Grande Guerre, mais aussi de sa stagnation entre 1914 et 1934. Autrement dit, la migration de masse du XIX ${ }^{\mathrm{e}}$ siècle a contribué à diminuer les inégalités salariales entre les pays.

13. Le contrôle des passeports devient obligatoire en 1919 en Allemagne et aux États-Unis, en 1920 en Grande-Bretagne.

14. De 300000 par an en 1850 , le nombre de migrants européens passe à 600000 en 1870 et à un million au début du siècle (Goldin et al., 2011 : 58). 
Les modèles formels sur la libéralisation de la mobilité de travail vont dans le même sens. Clemens (2011) a réalisé une revue de littérature sur les estimations des gains obtenus par l'ouverture des frontières. Ces gains conduiraient à augmenter le PIB mondial de $50 \%$ à $150 \%$, selon les hypothèses choisies ${ }^{15}$. Maintenir les frontières fermées nous fait perdre des milliers de milliards de dollars, nous alerte Clemens. Plus récemment, John Kennan (2013) estime que les gains de l'ouverture des frontières équivaudraient à ceux produits par une croissance miraculeuse qui aboutirait à doubler les revenus dans les pays en développement. Certaines études, plus pessimistes, arrivent à une estimation de ces gains à $17 \% \mathrm{du}$ PIB mondial, en déduisant les coûts psychologiques et monétaires incompressibles (Docquier, Machado et Sekkat, à paraître). Il s'agit d'une estimation «toutes choses égales d'ailleurs », qui ne prend pas en compte, par exemple, la manière dont les efforts politiques pourraient baisser certains coûts «incompressibles », dont certains relèvent de la discrimination et de la xénophobie. Mais quelles que soient leurs hypothèses, l'ensemble des modèles formels s'accordent à soutenir que la libéralisation de la mobilité de travail a des effets économiques bénéfiques.

À la lumière de ces études, l'augmentation de $3 \%$ de la migration de travail des pays pauvres vers les pays riches ne semble plus être une solution pauvreté-efficace. Si notre but est de lutter efficacement contre la pauvreté, la mesure qui maximise le nombre de personnes sorties de la pauvreté est la libre circulation. Il est possible que nous trouvions la maximisation trop ambitieuse, bien que nous répétions que notre but est «l'éradication » de la pauvreté. Il est possible que nous souhaitions l'éradication de la pauvreté à condition qu'elle n'implique pas l'ouverture des frontières. Mais le fait que la libre circulation des plus démunis soit une solution efficace si peu débattue indique un côté obscur de notre combat contre la pauvreté.

15. Les augmentations du PIB mondial vont de $67 \%$ (Iregui, 2005) ou 96,5\% (Moses et Letnes, 2004) à $122 \%$ (Klein et Ventura, 2009) ou 147,3\% (Hamilton et Whalley, 1984). 


\section{CONCLUSION}

Au moment où l'on réfléchit à l'agenda du développement qui succédera en 2015 aux objectifs du Millénaire, les recherches économiques sur la migration et son effet sur la pauvreté peuvent inspirer les décideurs. De telles recherches peuvent suggérer des objectifs simples, mesurables, comme l'augmentation de $3 \%$ de la migration de travail, notamment peu qualifiée, dans les pays riches. Étant donné le contexte politique, il est peu probable que les pays développés consentent à mentionner la migration dans l'agenda post-2015. Cependant, à la lumière des contributions des migrants du Sud qui représentent $2 \%$ de l'humanité et contribuent déjà quatre fois plus au développement que ne le font les États riches, le rôle de ceux-ci perd de son importance.

Le présent article s'ancre dans l'actualité du débat post-2015, sans pour autant se donner une visée pratique ou normative. Son but est d'étendre le critère de pauvreté-efficacité développé dans la littérature et d'en évaluer les conséquences logiques. En généralisant ce critère pour l'appliquer à une politique de développement plus complexe, on essaye de déterminer les situations où la migration doit se substituer à l'aide au développement et de les distinguer de celles où elle doit en être le complément. On prend appui sur deux littératures, l'une sur l'efficacité de l'aide, l'autre sur le rapport entre migration et développement, pour en conclure que si la migration doit toujours être un complément de l'aide, elle doit en fournir le substitut en cas d'inefficacité de l'aide, à côté d'autres mesures dont on a prouvé le succès dans la lutte contre la pauvreté.

\section{BIBLIOGRAPHIE}

Adams, Richard H., et John PAGE (2005), «Do international migration and remittances reduce poverty in developing countries? ?, World Development, vol. 33, n $^{\circ} 10$, p. $1645-1667$.

ADELMAN, Irma, et J. Edward TAYLOR (1990), «Is structural adjustment with a human face possible? The case of Mexico ", Journal of Development Studies, vol. $26, \mathrm{n}^{\circ} 3$, p. $387-407$

ALESINA, Alberto, et Béatrice WEDER (2002), «Do corrupt governments receive less aid?», The American Economic Review, vol. 92, n 4, p. 1126-1137. 
Baneue mondiale (1998), Assessing Aid: What Works, What Doesn't, and Why, Washington DC, World Bank.

Baneue mondiale (2006), Global Economic Prospects: Economic Implications of Remittances and Migration, Washington DC, World Bank.

Boone, Peter (1996), "Politics and the effectiveness of aid», European Economic Review, vol. 40, no 2, p. 289-329.

Burnside, Craig, et David Dollar (2000), «Aid, policies and growth », American Economic Review, vol. 90, no 4, p. 847-868.

Chenery, Hollis B., et Alan M. Strout (1966), "Foreign assistance and economic development", American Economic Review, vol. 66, $\mathrm{n}^{\circ} 4$, p. 679-753.

Clemens, Michael (2011), "Economics and emigration: Trillion-dollar bills on the sidewalk?", The Journal of Economic Perspectives, vol. 25, $\mathrm{n}^{\circ} 3$, p. 83-106.

Clemens, Michael, et Lant PritchetT (2008), «Income per natural. Measuring development of people rather than places", Population and Development Review, vol. 34, n 3, p. 395-434.

Collier, Paul (2008), The Bottom Billion. Why the Poorest Countries Are Failing and What Can Be Done About It, Oxford, Oxford University Press.

Collier, Paul, et David Dollar (2002), "Aid allocation and poverty reduction », European Economic Review, vol. 46, n 8, p. 1475-1500.

DocQuiER, Frédéric, Joël MACHADO et Khalid SEKKAT (à paraître), «Efficiency gains from liberalizing labor migration », [En ligne], [http://www.nottingham.ac.uk/gep/documents/conferences/2012/postgra duate-conference/joel-machado.pdf|.

Dumitru, Speranta (2009), «L'éthique du débat sur la fuite des cerveaux », Revue européenne des migrations internationales, vol. 25, $\mathrm{n}^{\circ} 1, \mathrm{p} .119-135$.

DURAND, Jorge, et al. (1996), «Migradollars and development : a reconsideration of the Mexican case», International Migration Review, vol. 30, $\mathrm{n}^{\circ}$ 2, p. 423-444.

EASTERLy, William ([2006] 2009), Le fardeau de l'homme blanc. L'échec des politiques occidentales d'aide aux pays pauvres, traduction française de P. Hersant et S. Kleiman-Lafon, Genève, Éditions Markus Haller.

FAINI, Ricardo (2002), «Développement, commerce international et migrations ", Revue d'économie du développement, vol. 16, nos 1-2, p. 85-116. 
Gaillard, Anne Marie, et Jacques Gaillard (1998), International Migration of the Highly-Qualified: A Bibliographic and Conceptual Itinerary, New York, Center for Migration Studies.

GalbRaiTH, John Kenneth ([1979] 1980), The Nature of Mass Poverty, Harvard: Harvard University Press, traduit en français par D. Blanchard, Paris, Gallimard.

Goldin, Ian, Geoffrey CAmeron et Meera Balarajan (2011), Exceptional People. How Migration Shaped Our World And Will Define Our Future, Princeton et Oxford, Princeton University Press.

GRIFFIN, Keith (1970), «Foreign capital, domestic savings and economic development ", Bulletin of the Oxford University Institute of Economics and Statistics, vol. 32, no 2, p. 99-112.

Guillaumont, Patrick, et Lisa Chauvet (2001), " Aid and performance: a reassessment », Journal of Development Studies, vol. 37, n 6, p. 66-87.

HAAS, Hein de (2010), «Migration and development: a theoretical perspective », International Migration Review, vol. 44, $\mathrm{n}^{\circ}$ 1, p. 227-264.

Hamilton, Bob, et John Whalley (1984), «Efficiency and distributional implications of global restrictions on labour mobility", Journal of Development Economics, vol. 14, no 1, p. 61-75.

Hatton, Timothy, et Jeffrey G. Williamson (2005), Global Migration and the World Economy. Two Centuries of Policy and Performance, Cambridge, MIT Press.

Hubbard, R. Glen, et William Duggan (2009), The Aid Trap. Hard Truths about Ending Poverty, New York, Columbia University Press.

IreguI, Anna Maria (2005), «Efficiency gains from the elimination of global restrictions on labour mobility ", dans George J. BORJAS et J. CRISP (dir.), Poverty, International Migration and Asylum, New York, Palgrave Macmillan, p. 211-238.

Islam, Muhammed N. (2005), "Regime changes, economic policies and the effects of aid on growth ", Journal of Development Studies, vol. 41, $\mathrm{n}^{\circ} 8$, p. 1467-1492.

JonEs, Richard C. (1998), « Remittances and inequality: A question of migration stage and geographic scale», Economic Geography, vol. 74, n 1, p. 8-25.

Kennan, John (2013), «Open borders », Review of Economic Dynamics, vol. 16, $\mathrm{n}^{\circ}$ 2, p. L1-L13. 
Klein, Paul, et Gustavo Ventura (2009), «Productivity differences and the dynamic effects of labor movements", Journal of Monetary Economies, vol. 56, no 8, p. 1059-1073.

LEVITT, Peggy (1998), « Social remittances: migration-driven local level forms of cultural diffusion", International Migration Review, vol. 32, $\mathrm{n}^{\circ} 4$, p. $926-948$.

Mann, Michael (1993), The Sources of Social Power. Vol II The Rise of Classes and Nation-States 1760-1914, New York, Cambridge University Press.

McGillivraY, Mark, et al. (2006), «Controversies over the impact of development aid: it works; it doesn't; it can but that depends ", Journal of International Development, vol. 18, $\mathrm{n}^{\circ}$ 7, p. 1031-1050.

Moses, Jonathon W., et Bjørn LeTNEs (2004), «The economic costs to international labor restrictions: Revisiting the empirical discussion", World Development, vol. 32, n 10 , p. 1609-1626.

Mosley, Paul (1980), «Aid, savings and growth revisited», Oxford Bulletin of Economics and Statistics, vol. 42, no 2, p. 79-95.

Moyo, Dambisa (2009), L'aide fatale. Les ravages d'une aide inutile et de nouvelles solutions pour l'Afrique, traduction française de A. Zavriew, Paris, JC Lattès.

Myrdal, Gunnar (1957), Rich Lands and Poor. The Road to World Prosperity, New York, Harper \& Brothers Publishers.

NuRKSE, Ragnar (1953), Problems of Capital Formation in Underdeveloped Countries, Oxford, Oxford University Press.

PAPANEK, Gustav F. (1973), «Aid, foreign private investment, savings and growth in less developed countries», Journal of Political Economy, vol. 81, $\mathrm{n}^{\circ} 1$, p. $120-130$.

PNUD (2009), Lever les frontières. Mobilité et développement humains, New York, Nations Unies.

PRITCheTT, Lant (2006), Let Their People Come: Breaking the Gridlock on Global Labor Mobility, Washington DC, Center for Global Development.

Rosenstein-Rodan, Paul Narcyz (1943), «Problems of Eastern and SouthEastern Europe industrialization », The Economic Journal, vol. 53, $\mathrm{n}^{\text {os }} 210$ 211, p. 202-211.

SIRKECI, Ibrahim, et al. (2012), Migration and Remittances during the Global Financial Crisis and Beyond, Washington DC, World Bank. 
Sachs, Jeffrey D. (2005), The End of Poverty. Economic Possibilities for our Time, New York, Penguin Books.

TAVAREs, J. (2003), «Does foreign aid corrupt?», Economic Letters, vol. 79, n 1 , p. $99-106$.

TAYLOR, L. (1990), «Foreign resource flows and developing country rowth: A three-gap Model. In problems of developing countries in 1990s", World Bank Discussion Paper 97, Washington, World Bank.

TORPEY, John (2000), L'invention du passeport. État, citoyenneté et surveillance, traduction française d'Elisabeth Lamoth, Paris, Belin.

Walmsley, Terry Louise, et L. Alan WinTERs (2003), «Relaxing the restrictions on the temporary movements of natural persons: A simulation analysis », Centre for Economic Policy Research Discussion Paper Series 3719, London, CEPR.

WHITE, Howard (1992), «What we know about aid's macroeconomic impact? An overview of the aid effectiveness debate», Journal of International Development, vol. 4, n 2, p. 121-137.

Winslow, Charles-Edward Amory (1951), «The cost of sickness and the price of health ", Monograph Series, n 7 , Genève, World Health Organization. 